\title{
Quasiclassical approach to the weak levitation of extended states in the quantum Hall effect
}

\author{
M. M. Fogler \\ School of Natural Sciences, Institute for Advanced Study, Olden Lane, Princeton, NJ 08540
}

(July 3, 2018)

\begin{abstract}
The two-dimensional motion of a charged particle in a random potential and a transverse magnetic field is believed to be delocalized only at discrete energies $E_{N}$. In strong fields there is a small positive deviation of $E_{N}$ from the center of the $N$ th Landau level, which is referred to as the "weak levitation" of the extended state. I calculate the size of the weak levitation effect for the case of a smooth random potential re-deriving earlier results of Haldane and Yang [Phys. Rev. Lett. 78, 298 (1997)] and extending their approach to lower magnetic fields. I find that as the magnetic field decreases, this effect remains weak down to the lowest field $B_{\min }$ where such a quasiclassical approach is still justified. Moreover, in the immediate vicinity of $B_{\min }$ the weak levitation becomes additionally suppressed. This indicates that the "strong levitation" expected at yet even lower magnetic fields must be of a completely different origin.
\end{abstract}

The levitation of the extended states in the quantum Halleffect has been proposed by Khmelnitskiil and Laughlin? (KL) almost fifteen years ago. Based on scaling arguments (see Ref. 3 for review) KL further suggested that the energies $E_{N}$ of the extended states can be obtained by solving the equation

$$
\sigma_{x y}\left(E_{F}=E_{N}\right)=(N+1 / 2) \frac{e^{2}}{2 \pi \hbar},
$$

where $\sigma_{x y}$ is the unrenormalized (short length scale) Hall conductance, $E_{F}$ is the Fermi energy, and $N$ runs through the set of integer values $N=0,1, \ldots$. Using the Drude-Lorentz formula

$$
\sigma_{x y}=\frac{e^{2}}{m} \frac{\omega_{c}}{\omega_{c}^{2}+\tau^{-2}} n\left(E_{F}\right),
$$

for the left-hand side, they obtained

$$
E_{N}=(N+1 / 2) \hbar \omega_{c}\left[1+\left(\omega_{c} \tau\right)^{-2}\right],
$$

where $\omega_{c}$ is the cyclotron frequency, $\tau$ is the zero-field momentum relaxation time, and $n$ is the electron density. Thus, in strong fields where $\omega_{c} \tau \gg 1$ the energy $E_{N}$ of the $N$ th extended state is close to the center of the $N$ th Landau level $E_{N}^{\infty}=\hbar \omega_{c}(N+1 / 2)$. As the magnetic field decreases, $E_{N}$ floats upward with respect to $E_{N}^{\infty}$, so that the relative deviation $\delta E_{N} / E_{N}^{\infty}$ increases

$$
\delta E_{N} / E_{N}^{\infty} \equiv\left(E_{N}-E_{N}^{\infty}\right) / E_{N}^{\infty}=\left(\omega_{c} \tau\right)^{-2},
$$

I will call the regimes $\delta E_{N} / E_{N}^{\infty} \ll 1$ and $\delta E_{N} / E_{N}^{\infty} \gg 1$ a "weak" and a "strong" levitation regimes, respectively. The regime of strong levitation is, of course, the most interesting. Unfortunately, this regime is also the hardest one to study. Up to date there is no progress in analytical treatment of this problem; as for the original arguments, they suffer from the absence pf completely satisfactory derivation of the scaling laws.
Not long ago, the study of the weak levitation phenomenon has been pioneered by Shahbazyan a ${ }^{2}$ d Raikh and then continued by several other groups. 10 The advances in the analytical treatment of the problem are due to the existence of a transparent physical picture of localization in sufficiently strong magnetic field and a smooth random potential.10 I will start with briefly recalling this picture, which leads to an approximate equality $E_{N} \approx E_{N}^{\infty}$. Then I will review the arguments of Haldane and Yange (HY) who identified the leading contribution to $\delta E_{N}=E_{N}-E_{N}^{\infty}$. Finally, I will present my own results.

The first simplification of the strong magnetic field limit comes from the approximate separation of the electron's motion into a fast rotation along the cyclotron orbit and a much slower dynamics of its guiding center. Unfortunately, the guiding center coordinates (unlike the coordinates of the particle itself) can not have definite values simultaneously. The characteristic uncertainty in the guiding center position is of the order of the magnetic length $l=\sqrt{\hbar / m \omega_{c}}$. And here the smoothness of the random potential brings the second crucial simplification: as long as the correlation length $d$ of the potential is much larger than $l$, this uncertainty can be ignored. As a result, there are two approximate integrals of motion: the energy of the cyclotron motion, i.e., the kinetic energy, and the energy of the guiding center degree of freedom, which is essentially the potential energy. In this approximation the guiding center is permanently bound to a certain level line $U(x, y)=$ const of the random potential $U(x, y)$ and performs a slow drift along such a line. The extended states correspond to unbounded level lines.10 In fact, for a wide class of potentials statistically symmetric under the sign change, the unbounded level line (percolation contour) is unique and is at zero energy 111 Since the total energy of the electron is equal to the sum of the energies of the cyclotron and the drift degrees of freedom, 
and the cyclotron energy is quantized in $\hbar \omega_{c}$ quanta, in this approximation $E_{N}$ is equal to $E_{N}^{\infty}$.

The calculation of the the weak levitation correction requires taking into account the Landau level mixing. HY demonstrated that such a mixing simply modifies the form of the potential in which the guiding center drifts. The new potential is not statistically symmetric under sign change. In fact, its percolation level is at higher energy.

Using the quantum-mechanical perturbation theory, HY found that

$$
\delta E_{N} \sim(N+1 / 2)\left(W^{2} / \hbar \omega_{c}\right)(l / d)^{4},
$$

where $W$ is the rms amplitude of the random potential. I retained only the first term in the perturbation series obtained by HY. The next term is smaller if $d \gg l N^{1 / 2}$. In addition, HY require that $\hbar \omega_{c} \gg W$. Denote by $R_{c}$ the classical cyclotron radius at energy $E_{N}$. It is easy to see that $R_{c}=\sqrt{2 N+1} l$, so that Eq. (5) becomes

$$
\delta E_{N} / E_{N}^{\infty} \sim\left(W / E_{N}\right)^{2}\left(R_{c} / d\right)^{4},
$$

and the condition $d \gg l N^{1 / 2}$ is simply $R_{c} / d \ll 1$. It immediately hits the eye that HY's result is expressed in terms of purely classical quantities, given the particle's energy is equal to $E_{N}$. Note also that the size of the effect is different from KL's formula (4). For instance, it is much larger provided that $R_{c} / d \gg W / E_{N}$. For a weak random potential, $W \ll E_{F} \simeq E_{N}$, this inequality can be met simultaneously with $R_{c} / d \ll 1$.

Naively, one might think that the weak levitation mechanism of $\mathrm{HY}$ stops functioning in lower magnetic fields where $R_{c} \gg d$. This turns out not to be true; however, the dependence of the weak levitation effect on magnetic field becomes slower,

$$
\delta E_{N} / E_{N}^{\infty} \sim\left(W / E_{N}\right)^{2}\left(R_{c} / d\right) \sim\left(\omega_{c} \tau\right)^{-1} .
$$

[The final expression follows from $\left.\tau \sim\left(d / v_{F}\right)\left(E_{F} / W\right)^{2}\right]$. Equation (7) is the central result of this paper. It is represented graphically in Fig. 1 together with the previous two. The plot should be understood as the dependence of the quantity $\delta E_{N} / E_{N}^{\infty}$ at the topmost Landau level on the ratio $R_{c} / d$. In other words, I assumed that the Fermi energy $E_{F}$ is fixed but the magnetic field is changing. For each value of the magnetic field one has to choose $E_{N}$ closest to $E_{F}$. Of course, discreteness of $N$ leads to some fine details on the curve in Fig. 1. Such details are insignificant for large $N$, which is assumed to be the case for the most points on the plot.

As one can see from Eq. (7), $\delta E_{N} / E_{N}^{\infty}$ monotonically grows as the magnetic field decreases. Naturally, one would like to know if it ever becomes of the order of one. The answer is negative: even at the lowest magnetic field $B_{\min }$ where the present approach is justified, the quantity $\delta E_{N} / E_{N}^{\infty}$ is still small. To verify that one needs to know what $B_{\text {min }}$ is. Clearly, $B_{\text {min }}$ is the largest of the two fields, at which the two simplifying considerations mentioned in the beginning of the paper breaks down. One is the field $B_{c}$ where the separation into the cyclotron and drift motion ceases to be valid, and the other is the field where the quantum uncertainty $(\sim l)$ of the guiding center position becomes of the order of $d$. The crossover field $B_{c}$ was calculated in Ref. 12. It corresponds to the point $R_{c} / d \sim\left(E_{F} / W\right)^{2 / 3}$ where the characteristic frequency of the drift motion becomes of the order of $\omega_{c}$. Combining this with the other condition, one obtains the largest value of the ratio $R_{c} / d$ where the calculation is still valid,

$$
\left(R_{c} / d\right)_{\max }=\min \left\{\left(E_{F} / W\right)^{2 / 3}, k_{F} d\right\}
$$

( $k_{F}$ is the Fermi wavevector in zero field). Substituting this value into Eq. (7) and keeping in mind that $W \ll E_{F}$, one obtains that $\left(\delta E_{N} / E_{N}^{\infty}\right)_{\max } \ll 1$ (see also Fig. 11).

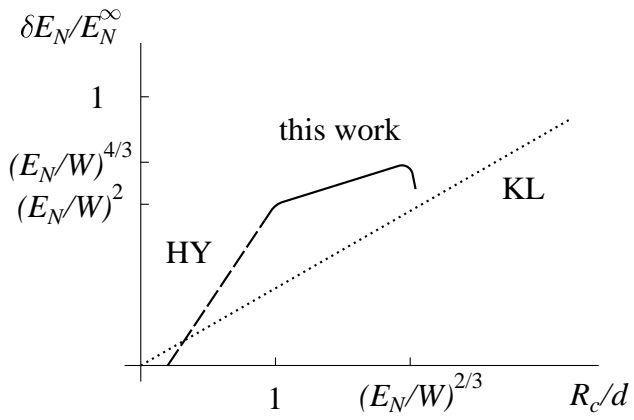

FIG. 1. The sketch of the dependence of the relative size of the weak levitation effect $\delta E_{N} / E_{N}^{\infty}$ at the topmost Landau level on the ratio $R_{c} / d$ (see text). Labels "HY," "this work," and "KL" refer to Eqs. (6), (1), and (4), respectively. Both axes are in log-scale.

Let us now turn to the derivation of Eqs. (6) and (7). I am going to show that the effect is completely classical and therefore the constraint $W \ll \hbar \omega_{c}$ imposed by $\mathrm{HY}$ is extraneous. The relevant condition is just $R_{c} / d \ll\left(R_{c} / d\right)_{\max }$. Also, in contrast to the quantummechanical treatment of Ref. 8, my instrument will be the classical perturbation theory. For this reason I will drop the subscript "F" in $E_{F}$. To ensure continuity with the previous paper 12 I will assume that the magnetic field is in the negative $\hat{z}$-direction, so that the cyclotron gyration is clockwise and the guiding center coordinates are given by

$$
\rho_{x}=x+\left(v_{y} / \omega_{c}\right), \quad \rho_{y}=y-\left(v_{x} / \omega_{c}\right),
$$

where $x$ and $y$ are the coordinate of the electron, and $\boldsymbol{v}=-v(\sin \theta, \cos \theta)$ is its velocity. The most convenient form of the equation of motion for $\rho$ is obtained if $U$ is re-expressed in terms $\boldsymbol{\rho}$ and $\theta$ only, i.e., as a new function

$$
V(\boldsymbol{\rho}, \theta)=U\left[\rho_{x}+\left(v / \omega_{c}\right) \cos \theta, \rho_{y}-\left(v / \omega_{c}\right) \sin \theta\right],
$$

where $v=\sqrt{2(E-V) / m}$ because of the energy conservation. The equation of motion for $\rho$ is 


$$
d \boldsymbol{\rho} / d \theta=\frac{1}{m \omega_{c}^{2}}\left[\hat{z} \times \nabla_{\rho} V(\boldsymbol{\rho}, \theta)\right] .
$$

This equation is of the Hamiltonian form with $\theta / \omega_{c}, \rho_{y}$, $m \omega_{c} \rho_{x}$, and $V$ being the time variable, the canonical coordinate, momentum, and the Hamiltonian function, respectively. The return to the original time variable can be accomplished by means of the equation

$$
\dot{\theta}=\omega_{c}+\hat{z}[\boldsymbol{v} \times \nabla U] /\left(m v^{2}\right) .
$$

It is convenient to expand $V(\boldsymbol{\rho}, \theta)$ in Fourier series,

$$
V(\boldsymbol{\rho}, \theta)=\sum_{k=-\infty}^{\infty} V_{k}(\boldsymbol{\rho}) e^{-i k \theta} .
$$

If $|k| \lesssim R_{c} / d$, the absolute value of $V_{k}$ is of the order of $W_{0}=W\left(d / R_{c}\right)^{1 / 2}$; otherwise, it is much smaller. Substituting Eq. (10) into Eq. (9), one obtains

$$
d \boldsymbol{\rho} / d \theta=\frac{1}{m \omega_{c}^{2}} \sum_{k=-\infty}^{\infty}\left[\hat{z} \times \nabla_{\rho} V_{k}(\boldsymbol{\rho})\right] e^{-i k \theta} .
$$

If one retains only the $k=0$ term, then the right-hand side does will not depend on $\theta$ and thus the guiding center motion will decouple from the cyclotron one. In this approximation the guiding center performs the drift along the level lines of the potential $V_{0}$. In the $R_{c} \ll d$ limit $V_{0}$ is very close to the original potential $U$ in agreement with the qualitative picture given above. If $R_{c}$ is larger than $d$, then quite a few $k \neq 0$ terms are of the same magnitude as $k=0$ one. In this case one can not simply ignore them; however, they can be made smaller by means of series of canonical (or almost canonical) transformations. 13 Each consecutive transformation reduces the oscillating terms by a factor of the order of $\gamma \equiv W_{0} /\left(m \omega_{c}^{2} d^{2}\right) \ll 1$. In the end they become suppressed by a factor $\exp (-$ const $/ \gamma)$. This program can be realized only if $\gamma \ll 1$. Equation $\gamma=1$ thus determines the magnetic field $B_{c}$ (see above) where the crossover from the adiabatic drift to the random walk of the guiding center occurs.12

For calculation of $\delta E_{N}$ to the first nonvanishing order in $\gamma$ only one such a transformation suffices. Let $p$ and $q$ be the new canonical coordinates after the transformation. Define the "renormalized" guiding center coordinates, $\rho_{x}^{(1)}=p /\left(m \omega_{c}\right)$ and $\rho_{y}^{(1)}=q$. It is easy to see that $\boldsymbol{\rho}^{(1)}$ has the following form

$$
\boldsymbol{\rho}^{(1)}=\boldsymbol{\rho}+\frac{1}{m \omega_{c}^{2}} \sum_{k \neq 0} \frac{1}{i k}\left[\hat{z} \times \nabla_{\rho} V_{k}\right]+O\left(\gamma^{2} d\right) .
$$

The $\theta$-independent term $V_{0}$ in the Hamiltonian function is transformed into $V^{\text {eff }}$ given by

$$
V^{\mathrm{eff}}=V_{0}+\frac{1}{m \omega_{c}^{2}} \sum_{k=1}^{\infty} \frac{\hat{z}}{i k}\left[\nabla V_{-k} \times \nabla V_{k}\right]+O\left(\gamma^{2} W_{0}\right) .
$$

Define

$$
U_{k}(\boldsymbol{\rho}, K) \equiv \oint \frac{d \phi}{2 \pi} e^{-i k \phi} U\left[\rho_{x}+R \cos \phi, \rho_{y}+R \sin \phi\right],
$$

where $K$ has the meaning of the kinetic energy and $R=\sqrt{2 K / m \omega_{c}^{2}}$ of the corresponding cyclotron radius. Note a useful formula

$$
\tilde{U}_{k}(\boldsymbol{q}, K)=i^{k} e^{-i k \theta_{k}} J_{k}(q R) \tilde{U}(\boldsymbol{q}),
$$

where tilde symbolizes the Fourier transform, $\boldsymbol{q}=$ $q\left(\cos \theta_{k}, \sin \theta_{k}\right)$, and $J_{k}$ is the Bessel function.

It is easy to see that

$$
\begin{aligned}
& V^{\mathrm{eff}}(\boldsymbol{\rho}, K)=U_{0}+\frac{1}{m \omega_{c}^{2}} \sum_{k=1}^{\infty}\left(Y_{k}-Z_{k}\right)+O\left(\gamma^{2} W_{0}\right), \\
& Y_{k}=\frac{\hat{z}}{i k}\left[\nabla U_{-k} \times \nabla U_{k}\right], \quad Z_{k}=\frac{1}{R} \frac{\partial}{\partial R}\left|U_{k}\right|^{2} .
\end{aligned}
$$

The obtained expression agrees with the effective potential of HY in the limit $R_{c} \ll d$. To see that one has to quantize the kinetic energy $K=E-V^{\text {eff }}=\hbar \omega_{c}(N+1 / 2)$ and keep only $k=1$ and $k=2$ terms, which dominate the sum in this limit. The levitation correction can be estimated 8 as

$$
\delta E_{N} \sim\left\langle V^{\mathrm{eff}}\right\rangle_{\mathrm{SP}}
$$

where "SP" stands for saddle-points, i.e., the points where

$$
\text { (A) } U_{0}^{x}=U_{0}^{y}=0, \text { (B) } U_{0}^{x x} U_{0}^{y y}-U_{0}^{x y} U_{0}^{y x}<0
$$

(the superscripts denote the partial derivatives). The rest of the paper is devoted to the derivation of Eq. (7) from Eqs. (14 16). I will assume that $U(x, y)$ is an isotropic Gaussian random potential with zero mean.

It turns out that for each $k, Y_{k}$ and $Z_{k}$ are correlated with at most one of the sets $\left\{U_{0}^{x}, U_{0}^{y}\right\}$ and $\left\{U_{0}^{x x}, U_{0}^{x y}, U_{0}^{y y}\right\}$. Therefore, each time one needs to calculate either $\left\langle Y_{k}\right\rangle_{A}$ or $\left\langle Y_{k}\right\rangle_{B}$, or simply the unrestricted average $\left\langle Y_{k}\right\rangle$ (and similarly for $Z_{k}$ ). The conditions " $A$ " and " $B$ " are given by Eq. (17). Notice that $Y_{k}$ and $Z_{k}$ are bilinear in $U$. This allows us to perform the $\langle\ldots\rangle_{A}$ averaging by means of the following general formula. Let $X_{1}$ and $X_{2}$ be linear in $U$, then

$$
\begin{aligned}
\left\langle X_{1} X_{2}\right\rangle_{A} & =\left\langle X_{1} X_{2}\right\rangle-\left\langle U_{0}^{x} U_{0}^{x}\right\rangle^{-1} \\
& \times\left(\left\langle X_{1} U_{0}^{x}\right\rangle\left\langle X_{2} U_{0}^{x}\right\rangle+\left\langle X_{1} U_{0}^{y}\right\rangle\left\langle X_{2} U_{0}^{y}\right\rangle\right) .
\end{aligned}
$$

Similarly,

$$
\begin{aligned}
& \left\langle X_{1} X_{2}\right\rangle_{B}=\left\langle X_{1} X_{2}\right\rangle+\left\langle U_{0}^{x x} U_{0}^{x x}\right\rangle^{-1}\left(\left\langle X_{1} U_{0}^{x y}\right\rangle\left\langle X_{2} U_{0}^{x y}\right\rangle\right. \\
& \left.-\frac{1}{2}\left\langle X_{1} U_{0}^{x x}\right\rangle\left\langle X_{2} U_{0}^{y y}\right\rangle-\frac{1}{2}\left\langle X_{1} U_{0}^{y y}\right\rangle\left\langle X_{2} U_{0}^{x x}\right\rangle\right) .
\end{aligned}
$$

Equations (18) and (19) can be obtained using general properties of Gaussian potentials and isotropicity of $U$. The $\langle\ldots\rangle_{A}$ averaging is needed for $Y_{2}$ and $Z_{1}$, the $\langle\ldots\rangle_{B}$ averaging is needed for $Y_{1}, Y_{3}$, and $Z_{2}$. All other $Y_{k}$ 's 
and $Z_{k}$ 's are to be averaged over the entire plane. The calculation is trivial but lengthy, and so I will give only the final result:

$$
\left\langle V^{\mathrm{eff}}\right\rangle_{\mathrm{SP}}=\frac{1}{m \omega_{c}^{2}}\left[\frac{15 A_{13}^{2}+A_{33}^{2}}{36 A_{04}}-\frac{A_{22}\left(2 A_{02}+A_{22}\right)}{4 A_{02}}\right],
$$

where $A_{k n}$ is defined as follows

$$
A_{k n}=\int \frac{d^{2} \boldsymbol{q}}{(2 \pi)^{2}} \tilde{C}(q) J_{0}(q R) J_{k}(q R) q^{n}
$$

with $C$ being the correlator $C(r)=\langle U(0) U(\boldsymbol{r})\rangle$. If $R_{c} \gg d$, then

$$
\left\langle V^{\mathrm{eff}}\right\rangle_{\mathrm{SP}} \simeq \frac{A_{02}}{4 m \omega_{c}^{2}} \sim \frac{W^{2}}{m \omega_{c}^{2} R d} .
$$

This concludes the derivation because Eqs. (16) and (21) immediately give Eq. (7).

The correction $\left(V^{\mathrm{eff}}-V_{0}\right)$ has quite peculiar properties: it is typically positive at the points where the gradient squared $\left(\nabla U_{0}\right)^{2}$ is smaller than its average value, typically negative otherwise, and almost vanishes on average. Indeed, one can show that the unrestricted spatial average of $V^{\text {eff }}$ is $\left\langle V^{\text {eff }}\right\rangle=-A_{01} /\left(2 m \omega_{c}^{2} R\right) \sim$ $-\left\langle V^{\text {eff }}\right\rangle_{\mathrm{SP}}\left(d / R_{c}\right)^{2}$, which is much smaller than $\left\langle V^{\mathrm{eff}}\right\rangle_{\mathrm{SP}}$ by the absolute value.

I speculate that the last property becomes important in the vicinity of $B_{c}$ where the crossover from the drift to the diffusion occurs. In the diffusive regime the trajectory of the guiding center is no longer bound to the level line $V^{\text {eff }}=$ const but samples the entire area. Thus $\left\langle V^{\mathrm{eff}}\right\rangle_{\mathrm{SP}}$ should approach $\left\langle V^{\mathrm{eff}}\right\rangle$. The latter is indistinguishable from zero within the accuracy of such an unrigorous argument. Thus, I expect the ultimate downfall of the solid curve in Fig. 1 near its termination point.

Concluding this paper, let us emphasize that the discrepancy between KL's formula (4) and Eqs. (6) and (7) does not contradict to Eq. (11), which comes from the scaling arguments. Indeed, for $B<B_{c}$ the "classical" or the "unrenormalized" Hall conductance is determined not by the average density $n$ but by the density $n_{p}$ near the percolation contour which is the area responsible for the transport; therefore,

$$
\sigma_{x y} \simeq \frac{e^{2}}{m \omega_{c}} n_{p}
$$

Equation (16) now follows from Eqs. (11), (22), and $n_{p}=\left(m / \pi \hbar^{2}\right)\left(E_{F}-V_{p}^{\text {eff }}\right)$ (" $p "$ again means "percolation"). At $B>B_{c}$ the percolation contour is of no importance so that Eq. (2) becomes valid, and presumably so does Eq. (4) as well.

In conclusion, I showed that the relative size $\delta E_{N} / E_{N}^{\infty}$ of the weak levitation effect is always much smaller than one. Moreover, it is expected to decrease near its termination point $B=B_{c}$. This strongly suggests that this effect has nothing to do with the stromg levitation predicted by Khmelnitskiil and Laughlin.
Finally, I should also mention that $\delta E_{N}$ can be measured experimentally by charting the global phase diagram of the quantum Hall effect,14 the procedure initiated by Glozman et al.5 Very interesting and puzzling findings have been reported recently.16 18 However, it seems that the electron-electron interaction plays a crucial role in the observed phenomena. This complicated issue is beyond the scope of the present paper.

This work is supported by the DOE Grant No. DEFG02-90ER40542, by the University of Minnesota's Doctoral Dissertation Fellowship, and by NSF Grant No. DMR-9616880. The idea for this work and some of the intermediate formulas have come to life in the course of collaboration with A. Yu. Dobin, V. I. Perel, and B. I. Shklovskii on Ref. 12. I thank them and also A. I. Larkin for useful discussions. I especially thank B. I. Shklovskii for comments on the manuscript.

${ }^{1}$ D. E. Khmelnitskii, Phys. Lett. A 106, 182 (1984).

${ }^{2}$ R. B. Laughlin, Phys. Rev. Lett. 52, 2304 (1984).

${ }^{3}$ B. Huckestein, Rev. Mod. Phys. 67, 357 (1995).

${ }^{4}$ T. V. Shahbazyan and M. E. Raikh, Phys. Rev. Lett. 75, 304 (1995).

${ }^{5}$ V. Kagalovsky, B. Horovitz, and Y. Avishai, Phys. Rev. B 52, R17 044 (1995).

${ }^{6}$ D. Z. Liu, X. C. Xie, and Q. Niu, Phys. Rev. Lett. 76, 975 (1996).

7 A. Gramada and M. E. Raikh, Phys. Rev. B 54, 1928 (1996).

${ }^{8}$ F. D. M. Haldane and Kun Yang, Phys. Rev. Lett. 78, 298 (1997).

${ }^{9}$ D. N. Sheng and Z. Y. Weng, Phys. Rev. Lett. 78, 318 (1997).

${ }^{10}$ M. Tsukada, J. Phys. Soc. Jpn. 41, 1466 (1976); S. V. Iordansky, Solid State Commun. 43, 1 (1982); R. F. Kasarinov and S. Luryi, Phys. Rev. B 25, 7626 (1982); S. A. Trugman, Phys. Rev. B 27, 1386 (1983).

${ }^{11}$ M. B. Isichenko, Rev. Mod. Phys. 64, 961 (1992).

${ }^{12}$ M. M. Fogler, A. Yu. Dobin, V. I. Perel, and B. I. Shklovskii, Phys. Rev. B 56, 6823 (1997).

13 See, for example, A. I. Neishtadt, Prikl. Mat. Mekh. 48, 197 (1984) [J. Appl. Math. Mech. 48, 133 (1984)].

${ }^{14}$ S. Kivelson, D. H. Lee, and S.-C. Zhang, Phys. Rev. B 46, 2223 (1992).

${ }^{15}$ I. Glozman, C. E. Johnson, and H. W. Jiang, Phys. Rev. Lett. 74, 594 (1995).

${ }^{16}$ S. V. Kravchenko, W. Mason, J. E. Furneaux, and V. M. Pudalov, Phys. Rev. Lett. 75, 910 (1995).

17 J. E. Furneaux, S. V. Kravchenko, W. E. Mason, G. E. Bowker, and V. M. Pudalov, Phys. Rev. B 51, 17 227 (1995).

18 S.-H. Song, D. Shahar, D. C. Tsui, Y. H. Xie, and D. Monroe, Phys. Rev. Lett. 78, 2200 (1997). 